\title{
Nivel de conocimiento sobre Diabetes Mellitus de los habitantes de Paso Ancho, San José Costa Rica, 2019
}

\author{
Knowledge Level about Diabetes Mellitus of the population of Paso Ancho, San José Costa Rica, 2019
}

\author{
Aurelia Blanco ${ }^{1}$ \\ Yazlin Alvarado ${ }^{2}$ \\ Catalina Capitán-Jiménez ${ }^{3}$
}

Fecha de recepción: 7-6-20

Fecha de aceptación: 16-3-21

\begin{abstract}
Resumen
Objetivos: La investigación busca conocer el porcentaje de personas que presentan un riesgo aumentado, así como el nivel de conocimiento de Diabetes Mellitus tipo 2 de la población de Paso Ancho, San José, Costa Rica. Metodología: Se aplicó el instrumento de factores de riesgo para el desarrollo de Diabetes Mellitus 2 de la American Diabetes Association (ADA) a 118 participantes de la Feria de Salud. Para evaluar el nivel de conocimiento sobre la enfermedad de la población en estudio se utilizó la versión en español del Diabetes Knowledge Questionnaire-24 (DKQ-24). Finalmente, se utilizó el programa de Microsoft Excel y SPSS para procesar estadísticamente los datos obtenidos en las encuestas e instrumentos. Resultados: El promedio del IMC fue de $27.9 \mathrm{Kg} /$ $\mathrm{m} 2$. Solo tres mujeres indican haber presentado diabetes gestacional durante su periodo de embarazo. Un $58 \%$ de los participantes tienen al menos un familiar que padece de diabetes, el 44\% de los presenta hipertensión arterial (HTA), de los cuales 15 son hombres y 37 mujeres. Y con respecto a la actividad física, un 53\% de las personas indican realizar actividad física, de los cuales 23 son hombres y 40 mujeres. Con relación a la evaluación del conocimiento sobre Diabetes Mellitus 2, se observa que ambos sexos tienen un conocimiento catalogado como no suficiente (90\%).

Palabras clave: diabetes mellitus 2, factores de riesgo, conocimiento, riesgo aumentado, obesidad.
\end{abstract}

\begin{abstract}
Objectives: The research seeks to know the percentage of people who present an increased risk, as well as the level of knowledge of Type 2 Diabetes Mellitus of the population of Paso Ancho, San José, Costa Rica. Methodology: The American Diabetes Association (ADA) instrument of risk factors for the development of Diabetes Mellitus 2 was applied to 118 participants of the Health Fair. The Spanish version of the Diabetes Knowledge Questionnaire-24 (DKQ-24) was used to assess the level of knowledge about the disease in the study population. Finally, the Microsoft Excel and SPSS program were used to statistically process the data obtained in the surveys and instruments. Results: The average BMI was $27.9 \mathrm{Kg} / \mathrm{m} 2$. Only three women indicate having had gestational diabetes during their pregnancy period. $58 \%$ of the participants have at least one family member who has diabetes, $44 \%$ of them have high blood pressure (hypertension), of which 15 are men and 37 are women. Regards to physical activity, $53 \%$ of people indicate physical activity, of which 23 are men and 40 are women. Regarding the evaluation of knowledge about Diabetes Mellitus 2, it is observed that both sexes have a knowledge classified as not enough (90\%).

Key words: diabetes mellitus 2, risk factors, knowledge, increased risk, obesity.

1 Licenciatura, Escuela de Nutrición y Consejo de Investigación, Universidad Hispanoamericana, Costa Rica, correo electrónico: aurelia. blanco@uh.ac.cr

2 Licenciatura, Escuela de Medicina, Universidad Hispanoamericana, Costa Rica, correo electrónico: yalvarado@uh.ac.cr

3 Maestría, Escuela de Nutrición y Consejo de Investigación, Universidad Hispanoamericana, Costa Rica, correo electrónico: ccapitan@ uh.ac.cr
\end{abstract}




\section{Introducción}

La Diabetes Mellitus (DM) tipo 2 supone entre el 85 y el $95 \%$ del total de casos de DM en el mundo, y se caracteriza por una resistencia a la insulina combinada con un déficit progresivo de producción de esta (Alonso et al., 2016; American Diabetes Association, 2017)usually leading to absolute insulin deficiency. $\mathrm{Al}$ mismo tiempo representa un desorden crónico en el metabolismo de la glucosa con serias consecuencias clínicas (Forouhi \& Wareham, 2019); Alonso et al. (2016) la definen como un grupo de trastornos metabólicos caracterizados por la hiperglucemia debida a defectos en la secreción o acción de la insulina. Este padecimiento se puede clasificar en cuatro categorías clínicas: tipo 1 (DM1), DM tipo 2 (DM2), DM gestacional y tipos de diabetes específicas debido a otras causas (American Diabetes Association, 2017).

El diagnóstico clínico de la DM2 se puede realizar cuando se supera el nivel de glucosa plasmática $126 \mathrm{mg} /$ dl o de $200 \mathrm{mg} / \mathrm{dl}$ tras dos horas de la ingesta de 75 $\mathrm{g}$ de glucosa; cuando las cifras de HbA1c en cualquier momento es mayor o igual $6.5 \%$ o cuando en presencia de síntomas de como poliuria, polidipsia y pérdida de peso encontramos una glicemia plasmática al azar mayor o igual a $200 \mathrm{mg} / \mathrm{dL}$ (Aschner M. et al., 2016) .

De acuerdo con el Ministerio de Salud de Costa Rica (2019) para el año 2014 la prevalencia de diabetes en la población general disminuyó $0.5 \%$ en comparación con la del año 2011. Sin embargo, datos de la Organización Mundial de la Salud (2020) indican que, en países de ingresos bajos o medios, la prevalencia de esta enfermedad va en aumento debido a la obesidad y a los cambios en los estilos de vida; según Jiménez-Montero y Villegas-Barakat (2021) SDMR, ischemic heart disease (IHD) la prevalencia de la DM2 en Costa Rica es similar a la descrita en los países industrializados. Esto no es sorprender si se observa que los índices de obesidad en Costa Rica son similares a los de Estados Unidos y Europa.

La OMS menciona que uno de los factores de riesgo más importantes para el desarrollo de DM2 es la obesidad, en Costa Rica según la Encuesta Nacional de Nutrición que se realizó entre los años 2008 y 2009, la prevalencia de sobrepeso y obesidad para las mujeres de 20-44 alcanzó el 59.7\% y para el grupo de 45 -64 años fue del $77.3 \%$. En el caso de los hombres de 20-64 años de edad, un 62.4\% fue categorizado con sobrepeso u obesidad (HasbumFernández, 2010). Sumado a esto, en Costa Rica en el periodo comprendido entre los años 2000 y 2004, en personas mayores de 20 años la incidencia acumulada de DM2 durante los 4 años fue del $4.77 \%$, con un promedio de $1.19 \%$ por año. La tasa de incidencia fue de 1.62 casos por 100 personas al año, e inclusive se mostraban tasas similares para ambos sexos (Hasbum-Fernández, 2010).

Para los años 2014-2018 se presentaron 8851 casos por año, con una tasa de 181 casos por 100 ooo habitantes para el territorio costarricense. Los datos indican que los cantones (entidad subnacional de segundo nivel según la división política de Costa Rica) con mayor tasa de incidencia fueron Acosta (tasa de 425.5 por cada 100 ooo habitantes), seguido de Palmares (tasa de 404.4) y en tercer lugar Parrita (tasa de 369.1). Los cantones con menor tasa promedio en el período fueron Alajuela, Talamanca y Atenas. Al mismo tiempo se indica que la incidencia aumenta a partir de los 40 años, se ve mayormente representada en el grupo de 65 a 69 años y principalmente se da en mujeres (Ministerio de Salud de Costa Rica, 2019).

Con respecto al nivel de conocimiento sobre diabetes mellitus 2; esta patología interfiere en el estilo de vida tanto de la persona que la padece como de su entorno familiar, por lo que una buena actitud frente a la enfermedad mejora la capacidad de enfrentamiento y esto es clave en la adopción y mantenimiento de determinados patrones de comportamiento como por ejemplo para la adopción de acciones de autocuidado que favorece la reducción del estrés, mejora al recibir el tratamiento, mejora de la autoestima y en el sentido de autoeficacia, además de una percepción más positiva sobre la salud (Martins Girotto et al., 2018).

Según el mismo autor el conocimiento sobre la enfermedad, se refiere al conjunto de informaciones que el paciente o familiar necesitan tener para administrar bien su condición de salud y constituye un aspecto fundamental del tratamiento de la patología. 
Su importancia es reconocida en diversos estudios realizados, independientemente de las características socioeconómicas y culturales (Martins Girotto et al., 2018).

Por lo tanto, la investigación busca conocer el porcentaje de personas que presentan un riesgo aumentado, así como identificar el nivel de conocimiento de Diabetes Mellitus tipo 2 de la población de Paso Ancho, San José, Costa Rica.

\section{Metodología}

El estudio presenta un tipo de diseño de investigación descriptivo en donde 118 personas que asistieron a la Feria de Salud organizada por la Universidad en una zona urbana de San José, Costa Rica accedieron a participar voluntariamente en la investigación. La primera parte de la encuesta consistía en un consentimiento informado en donde los participantes aceptaban o rechazaban participar en la investigación colocando su firma para así ser tomados en cuenta en el análisis de los datos, cumpliendo con todos los requerimientos descritos en la declaración de Helsinki.

A los mismos se les aplicó el instrumento de factores de riesgo para el desarrollo de Diabetes Mellitus 2 de la American Diabetes Association (ADA), esta consiste en 7 preguntas sencillas con un puntaje asignado que al sumarlo indica si la persona tiene un riesgo bajo (menor a 5) o aumentado (mayor o igual a 5) de presentar la enfermedad (ADA, 2018).

Para evaluar el nivel de conocimiento sobre la enfermedad de la población en estudio se utilizó la versión en español del Diabetes Knowledge Questionnaire-24 (DKQ-24), el cual tiene un índice de confiabilidad alfa de Cronbach de o.78. El cuestionario está conformado por 24 preguntas sobre conocimientos básicos de la enfermedad (10 ítems), control de la glucemia (7 ítems) y prevención de complicaciones ( 7 ítems). Las preguntas son de tipo cerrado con tres opciones de respuesta (sí, no y no sé). El nivel del conocimiento se clasifica en: suficiente (17 o más aciertos, equivalente al 70\% o más del total de aciertos) y conocimiento no suficiente (16 aciertos o menos).

Se utilizó el programa de Microsoft Excel y SPSS para obtener la estadística descriptiva (promedios, desviación estándar, distribuciones de frecuencias).

\section{Resultados}

En el estudio participaron 118 personas de las cuales el $36 \%$ corresponde al sexo masculino y el $64 \%$ al sexo femenino.

Tabla 1.

\section{Clasificación de participantes según sexo y edad.}

\begin{tabular}{|c|c|c|c|c|c|c|}
\hline \multirow{2}{*}{ Grupos de edades } & \multicolumn{2}{|c|}{ Masculino } & \multicolumn{2}{|c|}{ Femenino } & \multicolumn{2}{|c|}{ Total } \\
\hline & No. & $\%$ & No. & $\%$ & No. & $\%$ \\
\hline Menos de 40 años & 4 & $3 \cdot 4$ & 14 & 11.9 & 18 & $15 \cdot 3$ \\
\hline 40-49 años & 5 & 4.2 & 14 & 11.9 & 19 & 16.1 \\
\hline 50-59 años & 10 & 8.5 & 25 & 21.2 & 35 & 29.7 \\
\hline 60 años o más & 23 & $19 \cdot 5$ & 23 & $19 \cdot 5$ & 46 & 39.0 \\
\hline Total & 42 & 35.6 & 76 & $64 \cdot 4$ & 118 & 100.0 \\
\hline
\end{tabular}

Fuente: Elaboración propia, 2019. 
El promedio del IMC fue de $7.9 \pm 7.6 \mathrm{Kg} / \mathrm{m}^{2}$. De las 76 mujeres que participaron en el estudio, únicamente tres indican haber presentado diabetes gestacional durante su periodo de embarazo. Un $58 \%$ de los participantes tienen al menos un familiar que padece de diabetes, el $44 \%$ de los presenta hipertensión arterial (HTA), de los cuales 15 son hombres y 37 mujeres. Y con respecto a la actividad física, un $53 \%$ de las personas indican realizar actividad física, de los cuales 23 son hombres y 40 mujeres.

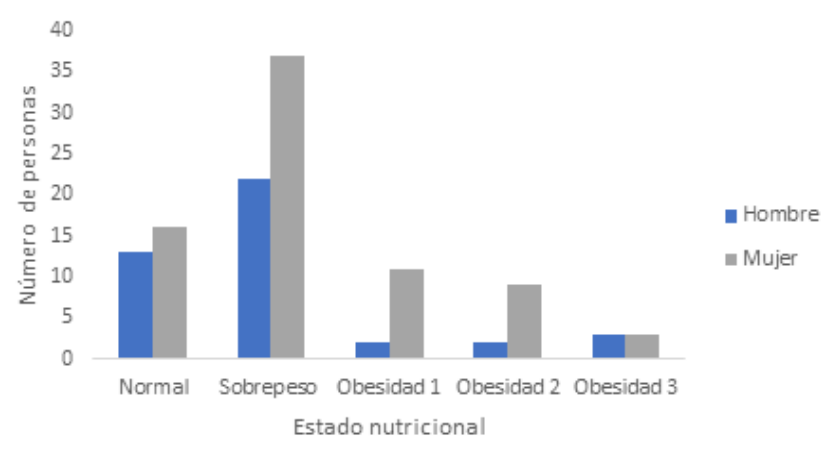

Figura 1. Clasificación del Estado Nutricional según IMC de la población de Paso Ancho, San José, 2019.

Fuente: Elaboración propia, 2019.

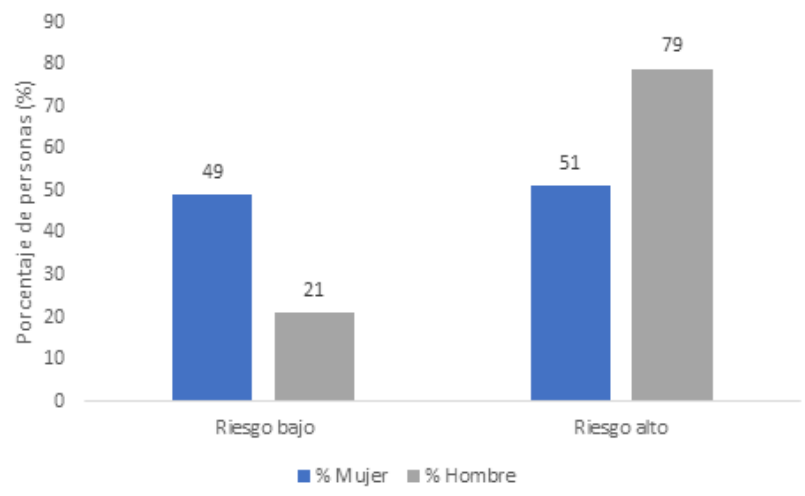

Figura 2. Puntaje de la American Diabetes Association para el riesgo de padecer diabetes Mellitus 2 de la comunidad de Paso Ancho, San José, 2019. $(\mathrm{P}<5$ : bajo riesgo, $\mathrm{P}>5$ alto riesgo).

Fuente: Elaboración propia, 2019.

Con respecto a la tabla 2, se puede ver que la mayoría de las preguntas (17 preguntas, $71 \%$ ) fue contestada correctamente por la mayoría de las personas, sin embargo, las preguntas 1, 12, 17 y de la 21 a la 24 fueron contestadas incorrectamente por la mayoría de las personas (7 preguntas, 29\%).

Tabla 2.

Porcentaje de respuesta correcta e incorrecta para las preguntas del cuestionario DKQ-24

\begin{tabular}{|c|c|c|c|c|c|}
\hline & Pregunta & $\begin{array}{l}\text { Respuesta incorrecta } \\
\text { No. }\end{array}$ & $\%$ & $\begin{array}{l}\text { Respuesta correcta } \\
\text { No. }\end{array}$ & $\%$ \\
\hline 1 & El comer mucha azúcar y otras comidas dulces es una causa de la diabetes & 107 & 91 & 11 & 9 \\
\hline 2 & $\begin{array}{l}\text { La causa más común de la diabetes es la fata de insulina que funciona } \\
\text { adecuadamente en el cuerpo }\end{array}$ & 24 & 20 & 93 & 79 \\
\hline 3 & $\begin{array}{l}\text { La diabetes es causada por que los riñones no pueden mantener el azúcar } \\
\text { fuera de la orina }\end{array}$ & 45 & 38 & 71 & 60 \\
\hline 4 & Los riñones producen insulina & 19 & 16 & 90 & 76 \\
\hline 5 & $\begin{array}{l}\text { En la diabetes sin tratamiento, la cantidad de azúcar en la sangre } \\
\text { usualmente sube }\end{array}$ & 8 & 7 & 109 & 92 \\
\hline 6 & Si yo soy diabético, mis hijos tendrán más riesgo de ser diabéticos & 15 & 13 & 103 & 87 \\
\hline 7 & Se puede curar la diabetes & 38 & 32 & 77 & 65 \\
\hline
\end{tabular}




\begin{tabular}{|c|c|c|c|c|c|}
\hline 8 & $\begin{array}{l}\text { Un nivel de azúcar de } 210 \text { en una prueba de sangre hecha en ayunas es } \\
\text { muy alto }\end{array}$ & 16 & 14 & 102 & 86 \\
\hline 9 & La mejor manera de controlar mi diabetes es con un examen de orina & 38 & 32 & 78 & 66 \\
\hline 10 & $\begin{array}{l}\text { Hacer ejercicio regularmente aumentará la necesidad de insulina o de } \\
\text { otro medicamento para mi diabetes }\end{array}$ & 25 & 21 & 86 & 73 \\
\hline 11 & $\begin{array}{l}\text { Hay dos tipos principales de diabetes: Tipo } 1 \text { (dependiente de insulina) y } \\
\text { Tipo } 2 \text { (no-dependiente de insulina) }\end{array}$ & 28 & 24 & 88 & 75 \\
\hline 12 & En un paciente diabético, comer mucho estimula la producción de insulina & 65 & 55 & 52 & 44 \\
\hline 13 & $\begin{array}{l}\text { Las medicinas son más importantes que la dieta y el ejercicio para } \\
\text { controlar mi diabetes }\end{array}$ & 37 & 31 & 77 & 65 \\
\hline 14 & La diabetes frecuentemente causa mala circulación & 17 & 14 & 101 & 86 \\
\hline 15 & Cortaduras y rasguños cicatrizan más despacio en diabéticos & 45 & 38 & 72 & 61 \\
\hline 16 & $\begin{array}{l}\text { Los diabéticos deberían poner cuidado extra al cortarse las uñas de los } \\
\text { dedos de los pies }\end{array}$ & 3 & 3 & 115 & 97 \\
\hline 17 & $\begin{array}{l}\text { Una persona con diabetes debería limpiar una cortadura primero con } \\
\text { yodo y alcohol }\end{array}$ & 81 & 69 & 36 & 31 \\
\hline 18 & $\begin{array}{l}\text { La forma como preparo mis alimentos es igual de importante que los } \\
\text { alimentos que ingiero }\end{array}$ & 13 & 11 & 105 & 89 \\
\hline 19 & La diabetes puede dañar mis riñones & 13 & 11 & 105 & 89 \\
\hline 20 & La diabetes puede causar que no sienta bien en mis manos, dedos y pies & 8 & 7 & 110 & 93 \\
\hline 21 & Son señales de azúcar alta en la sangre: El temblar y sudar & 90 & 76 & 28 & 24 \\
\hline 22 & Son señales de azúcar baja en la sangre: orinar seguido y tener mucha sed & 73 & 62 & 45 & 38 \\
\hline 23 & Las medias y las pantys elásticos apretadas son malas para los diabéticos & 97 & 82 & 21 & 18 \\
\hline 24 & Una dieta diabética consiste principalmente de comidas especiales & 102 & 86 & 16 & 14 \\
\hline
\end{tabular}

Fuente: Elaboración propia, 2019.

Con relación a la evaluación del conocimiento sobre Diabetes Mellitus 2, en la tabla 3 se observa que ambos sexos tienen un conocimiento similar del tema ya que más del 90\% de los hombres y las mujeres presentan un conocimiento catalogado como no suficiente. . El puntaje para el nivel de conocimiento sufiente fue de $17.4 \pm 0.6 \mathrm{y}$ para el nivel no suficiente fue de $13.7 \pm 1.9$.

Tabla 3.

\section{Nivel de conocimiento sobre Diabetes Mellitus 2}

\begin{tabular}{|l|l|l|l|l|l||l|}
\hline Nivel de conocimiento & Hombre & $\%$ & Mujer & $\%$ & Total & $\%$ \\
\hline No suficiente & 39 & 93 & 70 & 92 & 109 & 92 \\
\hline Suficiente & 3 & 7 & 6 & 8 & 9 & 8 \\
\hline Total & 42 & 100 & 76 & 100 & 118 & 100 \\
\hline
\end{tabular}

Fuente: Elaboración propia, 2019. 


\section{Discusión}

Los factores de riesgo de padecer Diabetes Mellitus 2 utilizando el instrumento proporcionado por la ADA, permitió conocer que existe un mayor riesgo en hombres con un $79 \%$ y un riesgo menor, aunque aumentado de $51 \%$ para las mujeres. Aunado a esto el conocimiento encontrado con respecto a los cuidados y especificaciones de la Diabetes Mellitus 2 es en su mayoría deficiente.

La mayoría de las personas que asistieron a dicha actividad presentaban un IMC de sobrepeso. Esto coincide con la FAO, que para el año 2018 indica que Costa Rica ocupa la sexta posición en Latinoamérica con respecto al porcentaje de obesos y que pasó a manejar un índice de obesidad del 34\% cuando hace 60 años poseía un índice de desnutrición del 50\% (Núñez, 2019).

Con respecto a la diabetes gestacional, de acuerdo con datos de la CCSS a partir del año 1997 se dieron registros de DMG para ese año las cifras de pacientes que presentaron la enfermedad fue de 531, en contraparte se indica que para el año 2015 fue de 1626 , lo que representa un aumento importante para la población femenina (Recio, 2016). Sin embargo, en este estudio solamente tres mujeres de las 76 entrevistadas afirmaron haber presentado esta enfermedad durante su embarazo.

En cuanto al alto porcentaje de hipertensos que se muestra en los resultados de esta investigación; un estudio de la Organización Mundial de la Salud y el Imperial College de Londres menciona que entre los años 1975 y el 2016 ha disminuido el padecimiento de esta enfermedad, sin embargo, las cifras son preocupantes al señalar que uno de cada cinco hombres padece de hipertensión. Por otro lado, Rodríguez (2017) menciona que Costa Rica se encuentra en la posición número 182 para hombres y 167 para mujeres de una lista de 200 países que fueron partícipes en dicho estudio. El país con mayor nivel de hipertensión para los hombres es Croacia con un 38.4\% y para las mujeres es Nigeria con un 35.8\%. El país con menor prevalencia es Corea del Sur con un 13.8\% para hombres y un $8.2 \%$ para mujeres (Rodríguez, 2017).
Al realizar el análisis de los datos relacionados a los factores de riesgo de padecer diabetes mellitus 2 utilizando el instrumento proporcionado por la ADA, se encontró un mayor riesgo en hombres con un $79 \%$ y un riesgo menor, aunque aumentado de 51\% para las mujeres. Lo anterior coincide con Laclé- Murray \& Valero-Juan (2008) quienes indican que un $72.3 \%$ de la población mayor a 20 años se encontraba en riesgo de padecer diabetes mellitus 2 en nuestro país según el mismo instrumento.

Al igual que en esta investigación, Carillo Alarcón (2015) indicaron que las preguntas con los puntajes más bajos estaban relacionadas con la etiología de DM, ya que los pacientes creían que se debía a un alto consumo de azúcar o alimentos dulces. Aunado a esto, el control metabólico, los signos y síntomas también resultaron dentro de las preguntas con menor conocimiento por parte de la población; esto es de riguroso cuidado ya que puede poner en riesgo la vida de la persona. Finalmente, con respecto a las complicaciones; al igual que el estudio de Carrillo Alarcón (2015) se observó una falta de conocimiento con respecto a este tema.

Para este estudio se encontró que el conocimiento de la mayoría de las personas se cataloga como deficiente. Lo anterior coincide con las investigaciones de Carrillo (2015), en donde encontraron que menos del 20\% de los pacientes contestaron de manera positiva las preguntas del cuestionario. Por otro lado, Arora et al. (2011), se encontraron que aunque los pacientes que padecen la enfermedad muestran mejores calificaciones que los familiares que se hacen cargo de ellos; ambos grupos exhibieron conocimientos pobres con respecto a las generalidades de la diabetes (Arora et al., 2020).

Similar que en esta investigación, los resultados de Saldaña, Aguayo, Hernández y Sánchez muestran promedios de respuestas adecuadas muy bajos en forma general; en especial en cuanto al conocimiento de la génesis y aspectos esenciales de la enfermedad; tal deficiencia es más notoria en los habitantes de lugares rurales, lo cual se puede explicar por la probable concepción de la etiología de la enfermedad (Saldaña et al., 2007). 
Por otro lado, un estudio en Perú mostró que la media de conocimientos fue de $12,9 \pm 4,8$; solo un $17,0 \%$ obtuvo un conocimiento bueno, y estuvo asociado con obtener información sobre diabetes de Internet y de otros pacientes. Asimismo, los puntajes positivos se relacionaron con tener un posgrado, tener más de 12 años de diagnóstico y una edad mayor a 70 años (Vicente Sánchez et al., 2010).

Finalmente se llega a la conclusión de que la prevalencia de riesgo de la población masculina es más alta (79\%) que la de mujeres (51\%) y que en general el nivel de conocimiento sobre la enfermedad es deficiente, esto aumenta la probabilidad de que cada vez más personas incidan en la patología. Por lo que es urgente capacitar y educar a las personas padezcan o no la enfermedad, tengan o no parientes que la padezcan ya que de una u otra manera se estaría educando para prevenir y mejorar los estilos de vida.

\section{Referencias bibliográficas}

Alonso, M., Santiago, A. M., Moreno, A., Carramiñana, F., López, F., Miravet, S., Seguí, M., Soriano, T., Pérez, M. P., Escríbano, J., Mancera, J., Comas, M., Barquilla, A., Gasull, V., \& Huidobro, C. (2016). Guías Clínicas: Diabetes Mellitus. EUROMEDICE.

American Diabetes Association. (2017). Classification and Diagnosis of Diabetes. Diabetes Care, 4o(Supplement 1), S11-S24. https://doi. org/10.2337/dc17-Soo5

Arora, S., Marzec, K., Gates, C., \& Menchine, M. (2020). Diabetes knowledge in predominantly Latino patients and family caregivers in an urban emergency department. Ethnicity \& Disease, 21(1), $1-6$.

Aschner M, Pablo; Muñoz V, Oscar Mauricio; Girón, Diana; Milena García, Olga; Fernández-Ávila, Daniel; Casas, Luz Ángela; Bohórquez, Luisa Fernanda; Arango T, Clara María; Carvajal, Liliana; Amanda Ramírez, Doris; Sarmiento, Juan Guillermo; Colon, Cristian Alejandro; Correa G, Néstor Fabián; Alarcón R, Pilar; Bustamante S, Álvaro, \& Andrés. (2016). Guía de práctica Clínica para la prevención, diagnóstico, tratamiento y seguimiento de la diabetes mellitus tipo 2 en la población mayor de 18 años. Colombia Médica, 47(2), 109-131.

Forouhi, N. G., \& Wareham, N. J. (2019). Epidemiology of diabetes. Medicine, 47(1), 22-27. https://doi. org/10.1016/j.mpmed.2018.10.004

Hasbum-Fernández, B. (2010). Epidemiología de la diabetes en Costa Rica. Avances en Diabetología, 26, 91-94.

Jiménez-Montero, J. G., \& Villegas-Barakat, M. (2021). Changes in diabetes mortality rate in Costa Rica 2007-2017. Diabetes Research and Clinical Practice, o(o). https://doi.org/10.1016/j. diabres.2021.108749 
Laclé-Murray, A., \& Valero-Juan, L. F. (2008). Incidencia de diabetes tipo 2 en un área urbano marginal de Costa Rica. Acta Médica Costarricense, 50(1), 29-34.

Martins Girotto, P. C., de Lima Santos, A., Silva Marcon, S., Martins Girotto, P. C., de Lima Santos, A., \& Silva Marcon, S. (2018). Conocimiento y actitud frente a la enfermedad de personas con diabetes mellitus atendidas en Atención Primaria. Enfermería Global, 17(52), 512-549. https://doi.org/10.6018/ eglobal.16.4.267431

Ministerio de Salud de Costa Rica. (2019). Análisis de la Situación de Salud 2018.

Nuñez, M. J. (2019). Obesidad es cada vez más frecuente en Costa Rica. Semanario Universidad. https:// semanariouniversidad.com/universitarias/ obesidad-es-cada-vez-mas-frecuente-en-costarica/

Organización Mundial de la Salud. (2020). Obesidad y sobrepeso. https://www.who.int/es/news-room/ fact-sheets/detail/obesity-and-overweight

Recio, P. (2016). Diabetes en ticas embarazadas se triplicó en últimos 18 años-La Nación. La Nación. https://www.nacion.com/ciencia/salud/diabetesen-ticas-embarazadas-se-triplico-en-ultimos-18anos/PH3EFBNA4VGJXJZMXKELEAPULI/story/

Rodríguez, I. (2017). Hipertensión en Costa Rica representa mayor riesgo para hombres que para mujeres. La nación. https://www.nacion.com/ciencia/salud/ hipertension-en-costa-rica-representa-mayorriesgo-para-hombres-que-para-mujeres/7EYW RXYIANH35 33 MFFOM2FX4EA/story/

Saldaña, R. B., Aguayo, L. Y. F., Hernández, G. L., \& Sánchez, E. (2007). Conocimientos usuales de los pacientes diabéticos en los estudiantes de medicina del occidente de México". 8.
Vicente Sánchez, B., Zerquera Trujillo, G., Rivas Alpizar, E., Muñoz Cocina, J., Gutiérrez Cantero, Y., \& Castañedo Álvarez, E. (2010). Nivel de conocimientos sobre diabetes mellitus en pacientes con diabetes tipo 2. MediSur, 8(6), 412-418. 